\title{
Exudates Detection in Retinal Images using Back Propagation Neural Network
}

\author{
Asha Gowda Karegowda \\ Siddaganga Institute of Technology, \\ Tumkur, India
}

\author{
Asfiya Nasiha \\ Siddaganga Institute Of Technology, \\ Tumkur, India \\ A.S .Manjunath \\ Siddaganga Institute Of Technology, \\ Tumkur, India
}

\author{
M.A.Jayaram \\ Siddaganga Institute Of Technology \\ Tumkur, India
}

\begin{abstract}
Exudates are one of the primary signs of diabetic retinopathy, which is a main cause of blindness and can be prevented with an early screening process. In this paper, authors have attempted to detect exudates using back propagation neural network. The publicly available diabetic retinopathy dataset DIARETDB1 has been used in the evaluation process. To prevent the optic disk from interfering with exudates detection, the optic disk is eliminated. Significant features are identified from the images after preprocessing by using two methods: Decision tree and GA-CFS method are used as input to the BPN model to detect the exudates and non-exudates at pixel level. The results prove that, BPN performance with features identified by Decision tree and GA_CFS approach has outperformed the performance of BPN with all inputs. The BPN classifier best performance was found with Sensitivity of $96.97 \%$, Specificity of $100 \%$ and classification accuracy of $98.45 \%$.
\end{abstract}

Keywords: Diabetic retinopathy, image preprocessing, back propagation neural network, exudates, HSI color space, features selection.

\section{INTRODUCTION}

Medical images analysis is a multidisciplinary research area, which covers image processing, machine learning pattern recognition and computer visualization. Retinal images are usually interpreted visually by the ophthalmologists in order to diagnose Diabetic Retinopathy (DR). Retinal image analysis system can be developed to assist ophthalmologists to make the diagnosis more efficiently. $\mathrm{DR}$ is the most common eye complication in diabetes. DR is globally the primary cause of visual impairment and blindness in diabetic patients. Screening of diabetic patients for early detection and timely treatment of diabetic eye disease significantly reduces the risk of vision loss. Diabetic Patients should have their eyes checked by an ophthalmologist at least once a year to see if retinopathy is present or progressing. These huge number of retinal images, need to be reviewed by the physicians, which is time consuming and costly. DR can lead to several retinal abnormalities, including micro aneurysms, hemorrhages, hard exudates and cotton wool spots. Hard exudates are yellowish intraretinal deposits, made up of serum lipoproteins. Exudates occur when lipid or fat leaks form abnormal blood vessel. If the exudates extend into the macular area, vision loss can occur [6, 18]. This paper investigates the application of back propagation neural network for detection of exudates in retinal images. Section
2 describes the related work of exudates detection using ANN. Section 3 briefs about the image preprocessing followed by feature extraction in section 4 . For the sake of completeness, the ANN classifier has been explained in section 5, and finally results and discussion in section 6 followed by conclusion in section 7 .

\section{RELATED WORK}

Akara et.al [1-4] have discussed comparative analysis of traditional and machine learning of exudates detection using mathematical morphology, fuzzy-c-means clustering, naïve Bayes Classifier, SVM, NN and, Nearest Neighbor (NN)classification. Fifteen features were identified out of which, finally six relevant features pixel's intensity value after preprocessing, the standard deviation of the preprocessed intensity, the pixel's hue, the number of edge pixels in a region around the pixel, the ratio between the size of the pixel's cluster and the size of the optic disc and DoG4 were selected using Naïve Bayes classifier and experimented with NN and SVM. Gardner et.al [9] has used neural network to identify the exudates in gray level images. Artificial neural network (ANN) was trained using back propagation method. The image was divided into squares of $30 \times 30$ and $20 \times 20$ pixel, based on which feature was being detected. The squares of gray-scaled images were classified by an expert observer into normal, blood vessel, exudates, hemorrhages or micro aneurysms. This information was used to train the ANN to classify the squares according to known outputs. Usher et.al [17] describes the development an automated system to detect abnormalities such as microneurysm, haemorrhages and exudates in color retinal images. They have used image processing to standardize color and contrast enhancement, segmentation to reveal lesions followed by classification of lesions using neural network. Jaykumari et.al [11] discusses the novel technique of intelligent segmentation followed by classification of exudates by using Echo State Neural Network (ECHO) Moving Window is used to obtain the statistical feature of the images (convex area, solidity, orientation and filled area), which are fed as input to ESNN. Osareh et.al [12] used fuzzy Cmean for segmenting the color retinal images, followed by classification of segmented regions into exudates and non-exudates using artificial neural network classifier. Further Osareh et.al has also compared the performance of $\mathrm{NN}$ for exudates detection with SVM [13] and three other classifiers: Linear Delta Rule (LDR), KNearest Neighbors (KNN) and Quadratic Gaussian classifier (QG) [14]. KNN performance was best among KNN, LDR and QG. 


\section{MATERIAL AND METHODS}

The publicly available diabetic retinopathy dataset DIARETDB1 has been used in the evaluation process. DIARETDB1 database contains a total of 89 images of resolution $1500 \times 1152$, with 47 of these images contain exudates while the remaining 42 either contain other type of lesions or are normal. Authors have used 10 images with 5 images with exudates and remaining 5 images without exudates for detecting exudates using pixel level classfication. The images have been scaled to 256 X256 size.

\subsection{Image Preprocessing}

Differences in luminosity, contrast and brightness inside retinal images make it complex to extort retinal features and make a distinction of exudates from other bright features in images. Hence Image Pre-Processing is required to eradicate noise present in the image and equalization of the irregular illumination associated with retinal images. The image preprocessing is explained below.

\subsubsection{HSI Color Space Conversion}

An RGB image is an $\mathrm{M} \times \mathrm{N} \times 3$ array of color pixels, where each color pixel is a triplet corresponding to red, green and blue components of RGB image at specified special location. Other color spaces or models include HSI, YcbCr, HSV, CMY, CMY and NISC color model $[5,15]$. As a first step of preprocessing the original RGB image is transformed to HSI (hue, saturation and intensity) color space. The HSI color space is commonly used color model for image-processing applications because it represents color similarly how the human eye senses colors. The other reason for selecting the HSI color space is that the Intensity matrix of the image can be separated from other components such as hue and saturation, so that significant information needed for the diagnosis of exudates, contained in the intensity matrix alone such as intensity, standard deviation of intensity, distance between optic disk mean pixel value and exudates and non-exudates pixels can be extracted. Hue is a color attribute that describes a pure color, Saturation gives a measure of the degree which the amount of white light mixed with the hue .The "intensity", "lightness" or "value" is related to the color luminance. The transformation equations used in the conversion of RGB to HSI are

$$
\begin{aligned}
& H=\theta(\text { ifB } \leq G) \\
& \text { else } 360-\theta \text { ifB }>G
\end{aligned}
$$

Where

$\theta=\cos ^{-1}\left\{1 / 2[(R-G)+(R+B)] /\left[(R-G)^{2}+(R-B)(G-B)\right]^{1 / 2}\right\}$

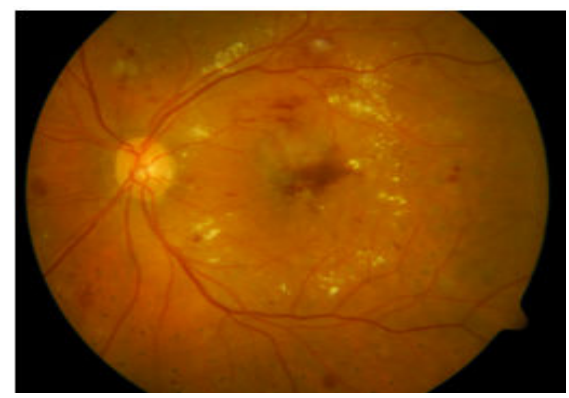

(a) RGB image
Saturation component is given by

$$
S=1-[3(R+G+B)][\min (\quad R, G, B)]
$$

Finally, the Intensity Component is given by

$$
I=1 / 3(R+G+B)
$$

\subsubsection{Median Filter}

Median filter is an effective method for suppressing isolated noise without blurring sharp edges. It replaces a pixel by the median of all pixels in the neighborhood of small sliding window. Median filter is an excellent choice for the removal of especially salt and pepper noise and horizontal scanning artifacts. As a part of image preprocessing, salt and pepper noise is added to intensity band and filtered by applying median filter of $3 \mathrm{X} 3$ size.

\subsubsection{Adaptive Histogram Equalization}

The fundus image usually have uneven illumination with areas at the center of the image brighter compared to sides and the brightness decreases as distance form the center of the image increases. To achieve uniform illumination adaptive histogram equalization is used so that the dark area in the input image becomes brighter in the output image bright area that was highly illuminated remains or reduces so that the image has uniform illumination.. The function adapthisteq() available in MATLAB is used for applying Contrast-Limited Adaptive Histogram Equalization to resultant Intensity band. The results of image preprocessing is shown below fig 1 .

\subsection{Optic Disk Elimination}

Since the optic disk has characteristics, bright intensity almost similar to hard exudates, the optic disk is detected and removed to reduce false positives. First the area of interest namely the optic disk is selected using Region of interest from the preprocessed intensity image I as I1. This is achieved using roipoly() function available in matlab. Further binary image of size 256x256 with all ones is taken say I2. I1 is subtracted from I 2 to get image I3. The image I3 is finally multiplied with preprocessed Intensity image I, which results in elimination of optic disk. The steps used for elimination of optic disk are shown in fig 2 .

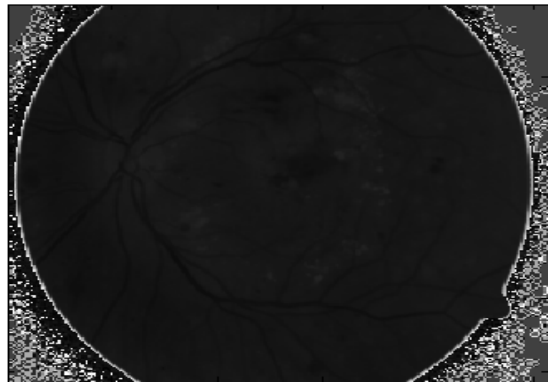

(b) Hue Image 


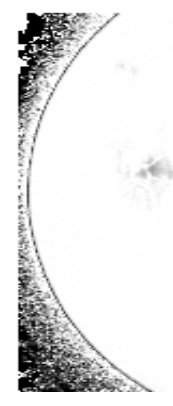

(c) Saturation Image

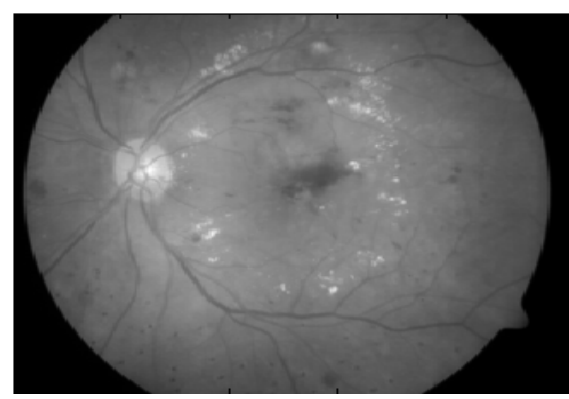

(d) Intensity Image

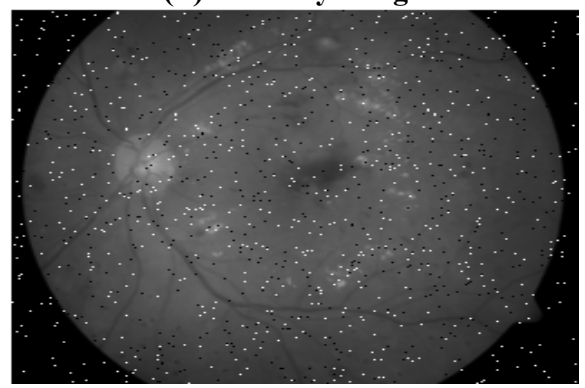

(e) Noise Image

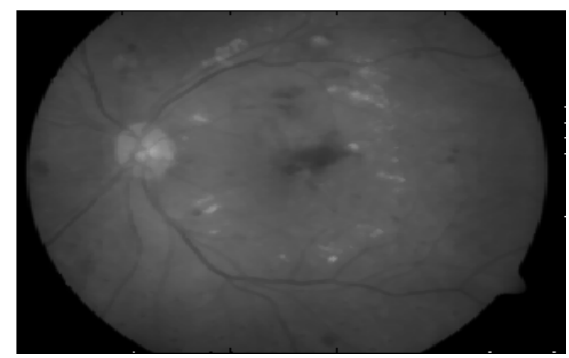

(f) Median Filter Image

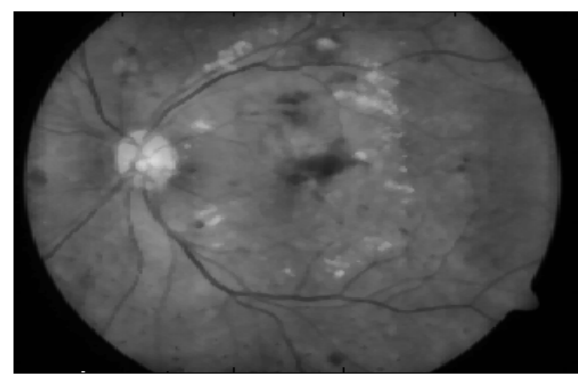

(g) CLAHE Image

Fig 1: Image Preprocessing (a) Original RGB Image (b) Hue Image (c) Saturation Image (d) Intensity Image (e) Noise Image (f) Median Filtered Image (g) Intensity Image after applying Contrast-Limited Adaptive Histogram Equilization.

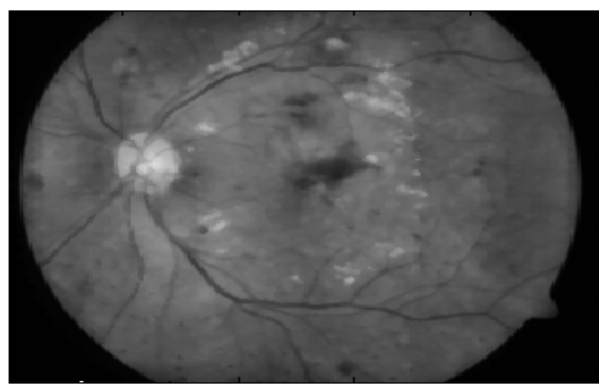

(a) Preprocessed Intensity Image (I)

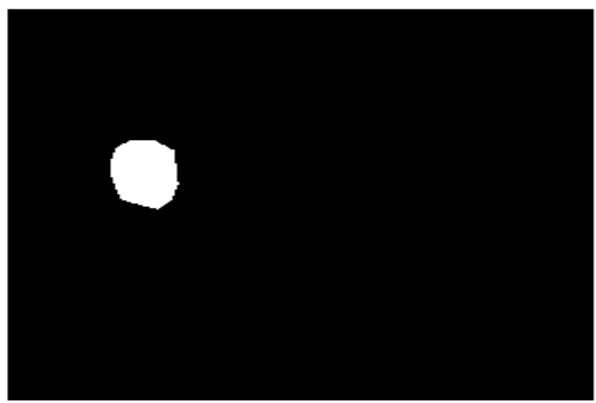

(b) I1 Image (Area Of Interest In White)

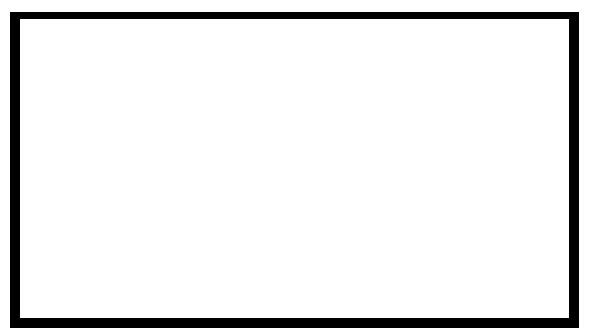

(c) I2 Image (With All Ones) 


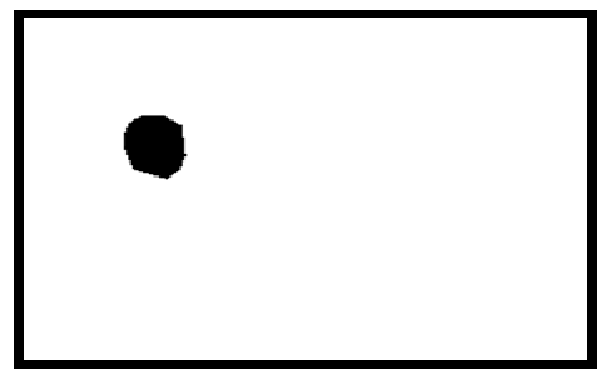

(d) I3 Image (I2-I1)

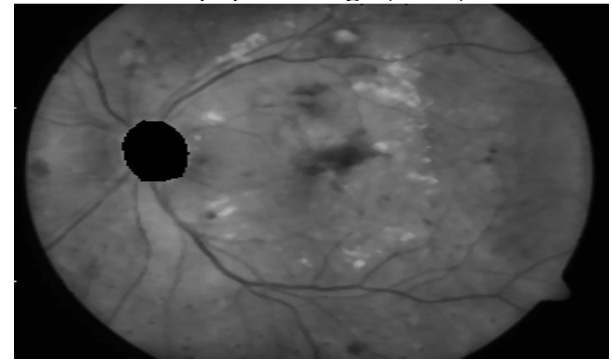

(e) Optic Disk Eliminated Image (I1*I3)

Fig 2: Optic Disk Elimination (a) Intensity Image after Preprocessing (b) Selection of Optic Disk using Region of Interest (c) Image with all Ones (d) Result of I2-I1 (e) Final Image with Optic Disk Eliminated

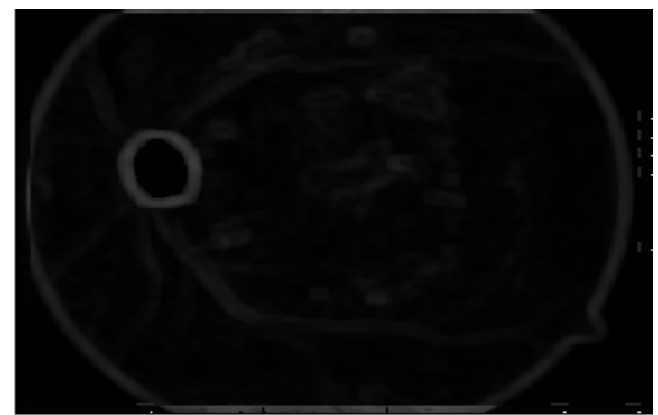

(a) Standard Deviation I Image

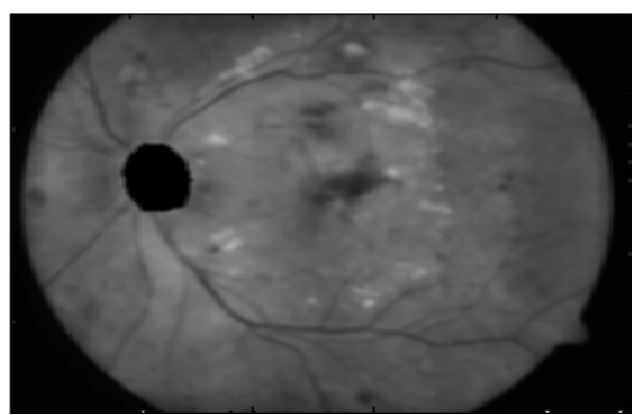

(b) MeanIntensity Image

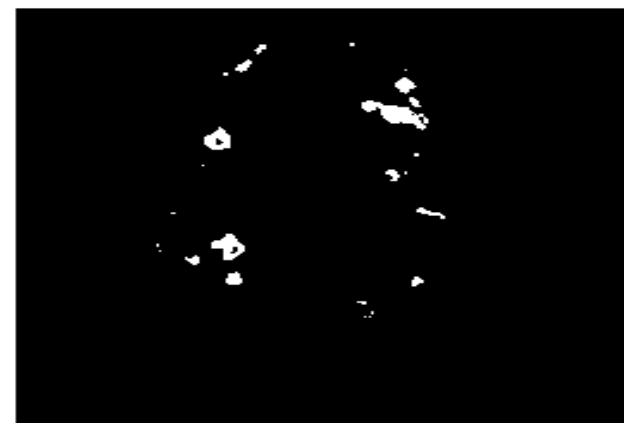

(c)Exudates Image

Fig 3: Exudates Features (a) Standard Deviation Intensity (b) Mean Intensity (c) Image showing Exudates after classification. 


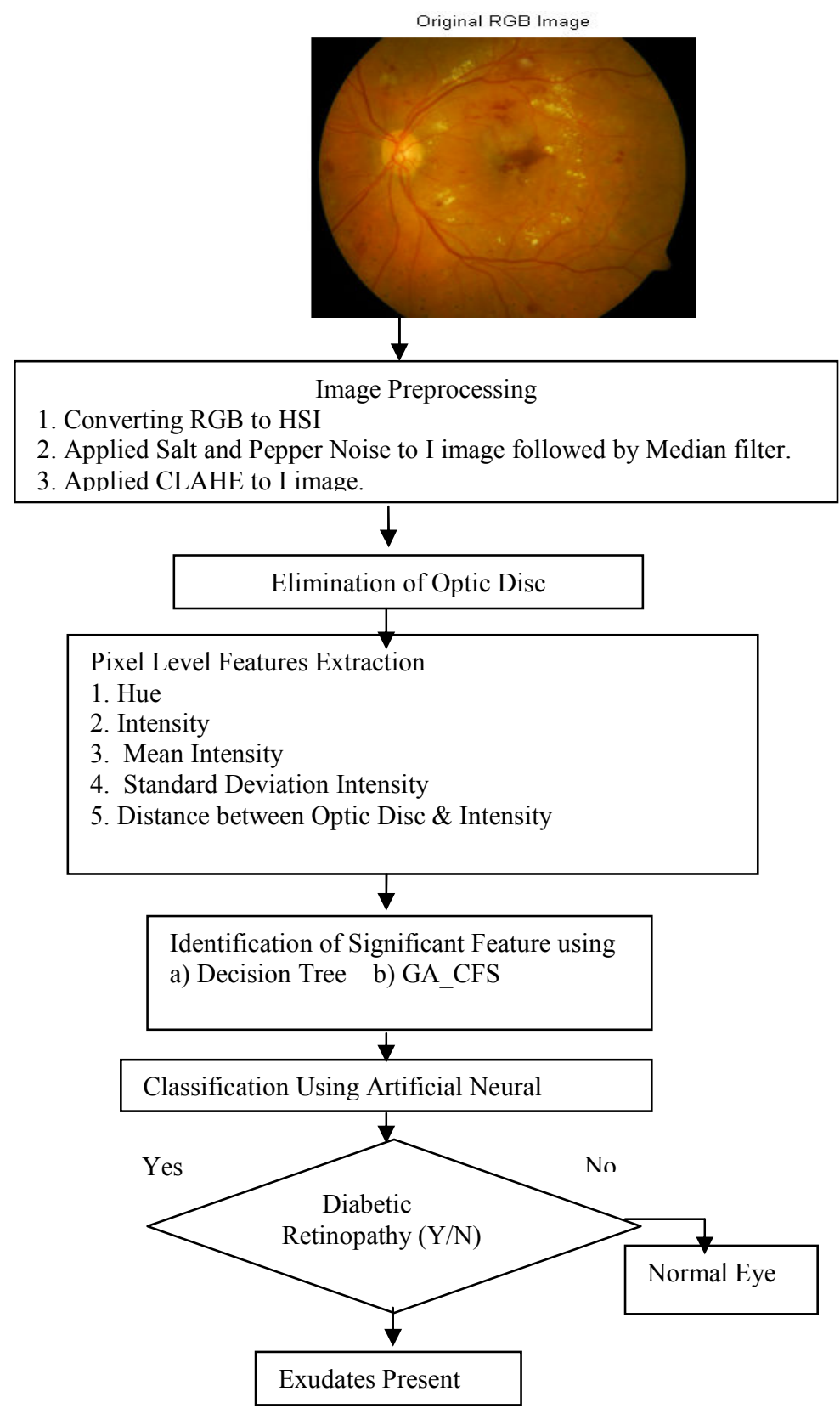

Figure 4: Method adopted for exudates detection

\section{FEATURE EXTRACTION}

Five pixel level features were selected as input for classfication of exudates and non-exudates pixels. Pixel Hue is extracted form Hue image ( Fig 1 (b)) that forms the chrominance information. Intensity matrix of the image separated from other components such as hue and saturation, after preprocessing, provides the remaining features needed for the diagnosis of exudates. The intensity of the image (Fig 1 (c), standard deviation values with window size $7 \times 7$ (Fig 3 (a)), distance between mean of optic disk pixels and pixels of exudates and non-exudates (using Euclidean distance), mean intensity (Fig 3(b)) using window size $3 \times 3$ were extracted from the preprocessed Intensity image. Since the exudates and optic disk have similar intensity, the Euclidean distance between optic disk pixels and exudates pixels will be less compared to that with nonexudates pixels. Hence the feature: distance between mean of optic disk pixels and exudates and non-exudates become one of the important features for exudates detection.

\section{CLASSIFICATION USING BACK PROPAGATION NETWORK}

Neural Network is an information-processing paradigm that is inspired by the way biological nervous systems, such as the brain, 
process information. The key element of this paradigm is the novel structure of the information processing system. It is composed of a large number of highly interconnected processing elements (neurons) working in unison to solve specific problems. Developing a neural network involves first training the network to carry out the desired computations. During the learning phase, training data is used to modify the connection weights between pairs of nodes so as to obtain a best result for the output nodes(s). The feed-forward neural network architecture is commonly used for supervised learning. Feed-forward neural networks contain a set of layered nodes and weighted connections between nodes in adjacent layers. Feed-forward networks are often trained using a back propagation-learning scheme. Back propagation learning works by making modifications in weight values starting at the output layer then moving backward through the hidden layers of the network. Neural networks have been criticized for their poor interpretability, since it is difficult for humans to interpret the symbolic meaning behind the learned weights. Advantages of neural networks, however, include their high tolerance to noisy data as their ability to classify patterns on which they have not been trained $[10,16]$.

\section{RESULTS AND DISCUSSION}

In this paper, five features: Hue, intensity of the image, standard deviation of intensity, distance between mean of optic disk pixels and pixels of exudates and non-exudates and mean intensity have been used inputs to ANN classifier for detecting the presence or absence of Exudates in the retinal images. 10 images, which were resized to $252 \times 252$ size are used for experimental evaluation. Training, Validation and Test data was prepared by manually collecting the feature for exudates and non-exudates pixels by equal partitioning method.

Feature subset selection is of great importance in the field of data mining. The high dimension data makes testing and training of general classification methods difficult. Feature selection is an essential pre-processing method to remove irrelevant and redundant data. It can be applied in both unsupervised and supervised learning. In supervised learning, feature selection aims to maximize classification accuracy. Authors have used two filters approaches namely Gain ratio and Correlation based feature selection for identifying relevant features. Decision tree is a simple tree like structure where non-terminal nodes represent tests on one or more attributes and terminal nodes reflect decision outcomes. The non-terminal nodes are taken as relevant features. In the first method adopted for attribute selection, the authors have used weka J4.8 to identify the significant attributes [7]. The J4.8 resulted in three relavant attributes namely Intensity, Std. Deviation of intensity and Distance between optic disk pixels and other pixels. In second method, the authors have used GA and Correlation based feature selection (CFS) in a cascaded fashion, where GA rendered global search of attributes with fitness evaluation effected by CFS. Genetic algorithm is used as search method with Correlation based feature selection as subset evaluating mechanism [8]. GA-CFS resulted in following four relevant attributes: Intensity, Std. Deviation of intensity, Hue and Distance between optic disk pixels. Experiments were conducted using different topologies of one hidden layer with 3 to 25 hidden nodes. Three different sets of inputs for the Neural network was experimented: (i) all inputs (ii) inputs identified by DT and (iii) inputs identified by GA_CFS methods. Number of epochs experimented was 10 to 40 epochs. The other network parameters were kept constant with step size for gradient descent as 0.1 , weight change momentum as 0.6 , and error tolerance as 0.01 .

Further Sensitivity, Specificity, Recall, and Precision and Fmeasure using true positive, true negative, false positive and false negative is computed for test data. For the sake of completeness few of the performance metrics have been discussed. True positive (TP) corresponds to the number of positive examples correctly predicted by the classifier. False negative (FN) corresponds to the number of positive examples wrongly predicted as negative by the classifier. False positive (FP) corresponds to the number of negative examples wrongly predicted as positive by the classifier. True negative (TN) corresponds to the number of negative examples correctly predicted by the classifier. The true positive rate (TP rate) or sensitivity is the fraction of positive examples predicted correctly by the model. TP Rate $=\mathrm{TP} /(\mathrm{TP}+\mathrm{FN})$. The false positive rate (FP rate) is the fraction of negative examples predicted as a positive class. FP Rate $=\mathrm{FP} /(\mathrm{TN}+\mathrm{FP})$.Precision is the fraction of records that actually turns out to be positive in the group the classifier has declared as a positive class. Precision $=\mathrm{TP}$ / $(\mathrm{TP}+\mathrm{FP})$. Recall is the fraction of positive examples correctly predicted by the classifier. Recall $=\mathrm{TP} /(\mathrm{TP}+\mathrm{FN})$.F-measure is used to examine the tradeoff between recall and precision. Fmeasure $=2 * \mathrm{TP} /(2 * \mathrm{TP}+\mathrm{FP}+\mathrm{FN})$.

The BPN classifier performance was measured using Sensitivity, Specificity, Recall, Precision and F-measure for test data with different topologies is shown in table 1 . Table 1 shows specificity and precision of $100 \%$ for ANN with (a) all inputs, (b) inputs identified by DT(c) inputs identified by GA_CFS. Further Sensitivity of the ANN classifier with inputs identified by both DT and GA_CFS is improved compared to sensitivity of ANN classifier with all inputs.

\section{CONCLUSION}

Early detection and timely treatment of DR can slow down the progression of the disease and avert blindness. Further with limited medical staff, an automated detection system can be used by nonexperts to indicate which patient requires referral to an ophthalmologist for additional investigation and treatment without further delay. The development of automated system can be effectively used as a filter of normal images, thereby reducing the burden on ophthalmologist, in addition to improvement of efficiency of diabetic screening program and reduction of cost. BPN classifier is successfully used to detect the exudates in retinal images. The BPN classifier performance used to detect exudates has been further improved by identifying significant features using two methods namely DT and GA_CFS method. Best performance of BPN was found with Sensitivity of $99.97 \%$ and Specificity of $100 \%$.

\section{REFERENCES}

[1] Akara Sopharak, K.T. New, Y.A. Moe, M.N. Dailey, and B Uyyanonvara, 2008. Automatic Exudate Detection with a naïve Bayes Classifier, Imaging in the Eye, IV, 2008, Https://www.cs.ait.ac.th

[2] Akara Sopharak, K.T. New, Y.A.; Moe, M.N. Dailey, and B Uyyanonvara. Automatic Exudates Detection us with a support vector machine classifier, Proc, International 
Conference on Embedded Systems and Intelligent Technology, pp.139-142, (2008).

[3] Akara Sopharak, Buyarit Uyyanonvara and Sarah Barman, Automatic Exudates Detection form Non-dilated Diabetic Retinopathy Retinal Images Using Fuzzy C-means Clustering, Sensors (2009), 9, 2148-2161.

[4] Akara Sopharak, Bunyarit Uyyanonvara, SarahBarman and Thomas Williamson. . Comparative Analysis of Automatic Exudates Detection between Machine Learning and Traditional Approaches, The Institute of electronic, Information and Communication Engineers (IEICE) Trans. Vol E92-D, N0 11, Nov (2009).

[5] Aibinu A.M., Iqbal M.I., Nilson M, and M.J.E. Salami, :Automatic Diagnosis of Diabetic Retinopathy From Fundus Images Using Digital Signal and Image Processing Techniques",Proceedings of the International Conference on Robotics, Vision, Information and Signal Processing, pp.510514, Penang, Malaysia, 28-30 November 2007.

[6] Asha Gowda Karegowda, Bharathi P.T, Jayaram M.A. ,Manjunath A.S, "Automatic Detection of Exudates in Diabetic Retinopathy using Traditional \& Machine Learning Techniques: An Overview", International Conference on Computing, New Delhi, 27-28 December 2010

[7] Asha Gowda Karegowda,M.A.Jayaram, " Integrating Decision Tree and ANN for Categorization of Diabetics Data", International Conference on Computer Aided Engineering, IIT Madras, Chennai, India,(2007).

[8] Asha Gowda Karegowda and M.A.Jayaram,"Cascading GA \& CFS for feature subset selection in Medial data mining", IEEE International Advance Computing Conference, Patiyala, India,(2009).

[9] Gardner, G.; Keating, D.; Williamson, T.; and Elliott, A. “ Automatic detection of diabetic retinopathy using an artificial neural network: a screening tool", British Journal of Ophthalmology, 80:940-944, (1996).
[10] Haykin, S.; Neural Networks- A comprehensive foundation, Macmillan Press, New York, (1994).

[11] Jayakumari, C.; and Santhanam, T. (2008). An Intelligent Approach to Detect Hard and Soft Exudates Using Echo State Neural Network, Information Technology Journal 7 (2), 386395, (2008).

[12] Osareh, A.; Mirmehdi, M.; Thomas, B.; Markham, R. (2002). Classfication and Localization of Diabetic-Related Eye Disease, A. Heyden et al(EDS).ECCV 2002, LNCS 2353,pp.502-516, (2002) .

[13] Osareh, A.; Mirmehdi, M.; Thomas, B.; Markham, R. "Comparative Exudates Classification using Support Vector Machine and Neural Networks", $5^{\text {th }}$ International Conference on Medical Image Computing-Assisted Intervention. Dohi, T.; Kikinis,(eds).PP, 413-420. September (2002)

[14] Osareh, A.; Mirmehdi, M.; Thomas, B.; Markham, R. "Automated Identification of Diabetic Retinal Exudates, in Digital Colour Images", British J. of Ophthalmology, vol. 87, no. 10, pp.1220--1223, Oct. (2003).

[15] Rafael C. Gonzalez and Richard E. Woods. 'Digital Image Processing using MATLAB', 2nd edition. Prentice Hall, 2002. ISBN 0-201-18075-8.

[16] Roy A., Artificial neural networks - a science in trouble, SIGKDD Explorations, 1:33-38,(2000)

[17] Usher, D.; Dumsky, M.; Himaga, M.; Williamson, T.H.; Nussey, Sl .; and Boyce, J." Automated detection of diabetic retinopathy in digital retinal images: a tool for diabetic retinopathy screening", Diametric Medicine, 21, 84-90, (2003).

[18] Facts About Diabetic Retinopathy, National Eye Institute http://www.nei.nih.gov/health/diabetic/retinopathy.asp

\begin{tabular}{|c|c|c|c|c|c|c|c|}
\hline $\begin{array}{c}\text { BPN } \\
\text { Topology }\end{array}$ & $\begin{array}{c}\text { Sensitivity } \\
(\%)\end{array}$ & $\begin{array}{c}\text { Specificity } \\
(\mathbf{\%})\end{array}$ & $\begin{array}{c}\text { FP } \\
\text { Rate }\end{array}$ & $\begin{array}{c}\text { Precision } \\
(\%)\end{array}$ & $\begin{array}{c}\text { Recall } \\
(\%)\end{array}$ & F-measure(\%) & $\begin{array}{c}\text { Classification } \\
\text { Accuracy (\%) }\end{array}$ \\
\hline $5-10-1$ & 94.12 & 100 & 0 & 100 & 94.12 & 96.97 & 96.94 \\
\hline $4-10-1$ & 96.97 & 100 & 0 & 100 & 96.97 & 98.47 & 98.45 \\
\hline $3-10-1$ & 96.97 & 100 & 0 & 100 & 96.97 & 98.47 & 98.45 \\
\hline
\end{tabular}

Table 1: BPN Performance for Exudates Classification using different Topologies 